\title{
Notificação de eventos: avanços e desafios no contexto da segurança do paciente
}

\author{
Notification of events: advances and challenges in the context of patient safety \\ Notificación de eventos: avances y desafíos en el contexto de la seguridad del paciente \\ Kellyne Quaresma Mourão1*, Amanda Maritsa de Magalhães Oliveira1.
}

\section{RESUMO}

Objetivo: Identificar os avanços quanto a notificação de eventos na assistência à saúde e analisar as fragilidades atreladas ao profissional de saúde. Métodos: Quanto as diretrizes metodológicas, trata-se de um estudo do tipo revisão bibliográfica, foram incluídos artigos dos anos de 2013 a 2017, idioma português e texto completo. Resultados: Consideraram-se os discutidos na revisão de literatura, permitindo considerar que a segurança do paciente emerge como uma forma de fortalecer o processo de trabalho e minimizar os danos que podem ocorrer durante a prestação do cuidado, ressaltando a importância da notificação de eventos. As fragilidades relacionadas à notificação de eventos adversos ainda permeiam a Segurança do paciente, o medo decorrente da cultura de segurança frágil e de ações punitivas, inviabiliza a notificação, fortalecendo a subnotificação. Considerações finais: A notificação surge como um elemento potencializador da Segurança do Paciente, contudo fragilidades ainda são identificadas, a cultura de segurança não é elemento predominante na mentalidade dos profissionais e a cultura punitiva, ainda, é um entrave.

Palavras-chave: Segurança do Paciente, Assistência ao paciente, Notificação.

\begin{abstract}
Objective: To identify the advances regarding the notification of events in health care and to analyze the fragilities linked to the health professional. Methods: Regarding the methodological guidelines, it is a study of the type bibliographic review, articles were included from the years 2013 to 2017, Portuguese language and full text. Results: We considered those discussed in the literature review, allowing us to consider that patient safety emerges as a way to strengthen the work process and minimize the damages that may occur during care delivery, stressing the importance of event notification. The weaknesses related to the notification of adverse events still permeate patient safety, the fear arising from the fragile safety culture and punitive actions, makes notification impossible, strengthening underreporting. Final considerations: Notification emerges as a potentiating element of Patient Safety, yet fragilities are still identified, the safety culture is not yet a predominant element in the mentality of professionals, and the punitive culture is still a hindrance.
\end{abstract}

Keywords: Patient Safety, Patient care, Notification.

\section{RESUMEN}

Objetivo: Identificar los avances en cuanto a la notificación de eventos en la asistencia a la salud y analizar las fragilidades vinculadas al profesional de la salud. Métodos: Las pautas metodológicas, es un estudio de los artículos de revisión de literatura tipo se incluyeron los años 2013 a 2017, el idioma portugués y el texto

${ }^{1}$ Faculdade Unyleya. Brasília - DF. *E-mail: kellynemourao@gmail.com 
completo. Resultados: Se consideraron los discutidos en la revisión de literatura, permitiendo considerar que la seguridad del paciente emerge como una forma de fortalecer el proceso de trabajo y minimizar los daños que pueden ocurrir durante la prestación del cuidado, resaltando la importancia de la notificación de eventos. Las fragilidades relacionadas con la notificación de eventos adversos aún permean la Seguridad del paciente, el miedo resultante de la cultura de seguridad frágil y de acciones punitivas, inviabiliza la notificación, fortaleciendo la subnotificación. Consideraciones finales: La notificación surge como un elemento potencializador de la Seguridad del Paciente, pero aún se identifican fragilidades, la cultura de seguridad no es elemento predominante en la mentalidad de los profesionales y la cultura punitiva, todavía, es un obstáculo.

Palabras clave: Seguridad del paciente, Asistencia al paciente, Notificación.

\section{INTRODUÇÃO}

A Portaria no 529 da Agência Nacional de Vigilância Sanitária (ANIVISA) institui o Programa Nacional de Segurança do Paciente (PNSP), programa importante por trazer em seu bojo a contribuição para qualificação do trabalho em saúde. O PNSP promove, apoia e implementa as iniciativas voltadas à segurança do paciente, através da implantação do Núcleos de Segurança do Paciente e gestão de riscos (ANVISA, 2013).

Os principais eventos adversos descritos pela literatura científica estão reacionados à: administração de medicamentos, vigilância do paciente, manutenção da integridade cutânea, recursos materiais (Duarte SCM, et al. 2015).

Miranda AP, et al. (2017) apontam a notificação de evento adverso como um prático meio de comunicação, haja vista que proporciona à instituição de saúde o conhecimento de fatos inesperados e indesejados. As notificações viabilizam o planejamento de processos mais seguros e permitem a prevenção de futuros eventos adversos. Desse modo, a comunicação deve ser oportuna, precisa, completa e livre de ambiguidade entre os membros da equipe de saúde.

Para Duarte SCM, et al. (2015) o reconhecimento dos eventos adversos e demais ocorrências também pode ser atrelado a cultura, crença e conhecimento dos profissionais acerca do problema, considerando que alguns profissionais têm dificuldades em perceber o erro. De acordo com Siman AG, et al. (2017) outro aspecto que dificulta o processo de notificação de evento adverso são as diferentes maneiras de agir dos profissionais de saúde frente ao ocorrido.

A notificação de eventos possibilita que a equipe que compõe o Núcleo de Segurança do Paciente, analise o ocorrido, realizando análise da causa raiz dos possíveis problemas que contribuíram para ocorrência do evento e propor medidas de melhoria atreladas aos processos de trabalho. Desta forma, a prestação do cuidado se torna mais segura, a medida que os processos também se tornam.

Embora seja indubitável a importância da notificação, muitas fragilidades ainda permeiam esse processo. Neste sentido, faz-se necessário identificar as fragilidades existentes no processo de notificação. $O$ estudo é relevante, uma vez que o tema segurança do paciente é recente e de extrema importância para saúde no Brasil, ainda há necessidade de mais publicações sobre o tema.

Ressalta-se também que o estudo trará contribuições para a comunidade acadêmica, pela discussão realizada, assim como trará benefícios para a população, pois os resultados poderão suscitar planos de ação em instituições de saúde, de forma a fortalecer os processos de trabalho, impactando positivamente na prestação do cuidado.

Sendo assim, o objetivo do presente estudo é Identificar os avanços quanto a notificação de eventos na assistência à saúde e analisar as fragilidades atreladas ao profissional de saúde no processo de notificação de eventos. 


\section{MÉTODOS}

O estudo trata-se de uma pesquisa bibliográfica, realizada por meio de fontes secundárias tais como artigos científicos da área saúde, abordando o tema de interesse. A pesquisadora trabalha a partir de contribuições dos autores dos estudos analíticos constantes nas fontes pesquisadas. $O$ universo bibliográfico abrangeu artigos científicos, disponibilizados em bancos de dados científicos: Biblioteca Virtual de Saúde (BVS); Scientific Electronic Library Online (SCIELO). Os descritores utilizados para realização da pesquisa foram: Segurança do Paciente; Assistência ao paciente; Notificação. Aplicou-se os seguintes critérios de inclusão: produções disponíveis online, formato de artigos científicos completos, produções relacionadas à temática em questão, idioma em português, compreendendo o período de 2013 a 2017. Foram excluídos do presente estudo os artigos que não atenderam aos critérios acima utilizados.

\section{RESULTADOS E DISCUSSÃO}

\section{A relevância da Segurança do Paciente}

A segurança do paciente é uma preocupação antiga em âmbito mundial, em se tratando da prestação dos cuidados. Não obstante, no Brasil ainda é um tema recente e muitas instituições de saúde estão se adequando a essa realidade. A segurança do paciente, ao longo de sua construção e consolidação, surge como um elemento potencializador, no que tange a melhoria dos processos de trabalho e redução das falhas durante a prestação do cuidado.

Segundo Fernando FSL, et al. (2016) o âmago da segurança do paciente é a preocupação com a magnitude da ocorrência dos eventos adversos, e possíveis danos ocasionados ao paciente através do cuidado de saúde.

Reis CT, et al. (2013) abordam que a preocupação com a segurança aumenta de forma proporcional a complexidade dos sistemas de prestação de cuidados. Retratam os eventos adversos passíveis de ocorrência nos serviços de saúde, e a relevância dos mesmos, pois a maioria se refere a eventos evitáveis.

\section{Avanços atrelados à notificação de eventos na assistência à saúde}

A notificação surge como um elemento potencializador da Segurança do Paciente, haja vista que a partir do ocorrido será realizada uma análise na causa raiz, as fragilidades do processo serão elencadas e ações serão traçadas para fortalecer o processo de trabalho, agindo, dessa forma, tanto na segurança do paciente quanto na segurança do profissional.

Partindo da perspectiva que a partir da ocorrência de um evento medidas devem ser tomadas para que um evento da mesma natureza não ocorra, evidencia-se a importância da notificação dos eventos na assistência à saúde.

O cuidado prestado aos pacientes é complexo e requer manejo de qualidade, de forma a não ocasionar danos desnecessários ao indivíduo. No ambiente hospitalar, a terapia medicamentosa é amplamente utilizada para tratamento das doenças e manutenção da saúde. Neste âmbito, os pacientes hospitalizados e que fazem uso de múltiplos medicamentos encontram-se mais vulneráveis à ocorrência de eventos adversos (OLIVEIRA RM, et al. 2014).

As questões relacionadas à segurança do paciente constituem um problema de saúde em nível mundial. Logo, os riscos e a ocorrência de eventos que ocasionam danos ao paciente têm aumentado em todos os ambientes, em especial em âmbito hospitalar. Nas últimas décadas, nota-se uma preocupação em torno das políticas, para a melhoria da qualidade assistencial e a problemática que envolve os riscos à segurança do paciente, torna-se tema de vários estudos no sistema de saúde (SILVA AT, et al. 2016).

Nota-se que a notificação de eventos adversos já vem sendo apontada nos estudos como uma medida preventiva para ocorrência de erros na assistência à saúde. Há ações positivas da assistência de enfermagem na segurança do paciente como a implantação de protocolos de assistência, o boletim de 
notificação de eventos adversos, o uso do checklist da cirurgia segura e utilização dos diagnósticos de enfermagem na redução de riscos. (SILVA AT, et al. 2016).

A notificação de evento adverso é um prático meio de comunicação, pois proporciona à instituição de saúde o conhecimento de fatos inesperados e indesejados. As notificações viabilizam o planejamento de processos mais seguros e permitem a prevenção de futuros eventos adversos. Desse modo, a comunicação deve ser oportuna, precisa, completa e livre de ambiguidade entre os membros da equipe de saúde (MIRANDA AP, et al. 2017).

Apesar das dificuldades encontradas no processo de notificação de EA, verificou-se a importância de notificar, a notificação foi referida como fonte de informações úteis para melhorar a assistência ao paciente (SIMAN AG, et al. 2017)

A notificação foi apontada como instrumento de auxílio à gestão do cuidado à saúde, identificação de problemas e busca de alternativas para solucionar os problemas da assistência relacionado a aos profissionais da saúde (PAIVA MCMS, et al. 2014).

É importante que as instituição de saúde possuam ferramenta específica para realizar as notificações e também promover a motivação, por parte da equipe de saúde, na alimentação dessa ferramenta (CHAGAS KD e SELOW MLC, 2016).

Neste sentido, nos estudos abordados foi identificando a potencialidade dessa ferramenta na melhoria dos processos e, dessa forma, como instrumento fundamental para Segurança do Paciente na prestação dos cuidados.

\section{Fragilidades atreladas ao profissional de saúde relacionado ao contexto da segurança do paciente}

Em se tratando da notificação de eventos algumas fragilidades ainda são identificadas, ressalta-se que a cultura de segurança ainda não é elemento predominante da mentalidade dos profissionais e por vezes permeia o medo por conta da cultura punitiva.

O erro ainda é um termo forte, pois dependendo da magnitude do mesmo o paciente será pouco ou muito prejudicado. Lembrando que os eventos não incidem somente sobre paciente, mas também no profissional prestador do cuidado.

A segurança do paciente, na atualidade, suscita melhorias atreladas aos processos de trabalho e é uma temática extremamente relevante em se tratando do âmbito hospitalar, visando sempre à prestação do cuidado de forma segura. Observou-se os avanços da notificação dos eventos adversos, visto que nos estudos abordados foi identificando a potencialidade dessa ferramenta na melhoria dos processos e instrumento fundamental para Segurança do Paciente na prestação dos cuidados. A notificação de eventos adversos já vem sendo apontada como uma medida preventiva para ocorrência de erros na assistência á saúde.

O reconhecimento dos eventos adversos e demais ocorrências também pode ser atrelado a cultura, crença e conhecimento dos profissionais acerca do problema, considerando que alguns profissionais têm dificuldades em perceber o erro. As principais causas atreladas a ocorrência de eventos adversos estão os fatores inerentes ao déficit de pessoal, sobrecarga de trabalho, problemas de relacionamento entre a equipe multiprofissional, dentre outros (DUARTE SCM, et al. 2015).

A existência de baixo conhecimento dos profissionais de saúde sobre eventos adversos e como notificálos, medo dos profissionais de saúde em expor os erros devido à política de punição das instituições e baixa adesão da técnica de higienização das mãos (SILVA AT, et al. 2016).

Infelizmente em algumas instituições de saúde o evento adverso devidamente registrado, caracterizando a notificação do evento, incide em punição ao profissional e saúde, inclusive demissão. Essa fragilidade culmina na subnotificação de eventos e, consequentemente, em processos frágeis. 
O posicionamento do profissional de enfermagem irá ocorrer de acordo com a cultura institucional. Para enfermeiros e técnicos de enfermagem o erro não ocorre de forma intencional, e muitas vezes o profissional não identifica a ocorrência. Foi apontado como reconhecimento a impossibilidade de manter a atenção centrada na atividade executada (DUARTE SCM, et al. 2015).

Outro aspecto que dificulta o processo de notificação de EA são as diferentes maneiras de agir dos profissionais de saúde frente ao EA, mesmo constando o passo a passo da notificação em procedimento operacional padrão. Foram identificadas, também divergentes formas de comunicar o evento adverso pelos técnicos de enfermagem: comunicação aos médicos ou enfermeiros, anotações em prontuário ou livro de ocorrência, ou não sabem o que fazer (SIMAN AG, et al. 2017).

Os enfermeiros são mais propensos a relatar incidentes do que os médicos, dentre os motivos foram elencados, pelos médicos, limitação de tempo, incerteza sobre o que relatar, expectativa de punição ou culpa e percepção de que a notificação de incidentes possa não culminar em melhorias (CAPUCHO HC, et al. 2013).

A notificação e discussão dos erros ocorridos ainda estão aquém frente às organizações hospitalares, em que os grupos envolvidos não notificam um erro, por medo de punições ou desconhecimento sobre o tema (CHAGAS KD e SELOW MLC 2016).

Faz-se necessário a implantação de ações educativas, juntamente às medidas que respaldem os profissionais em relação à ocorrência dos eventos adversos, concomitante a isso estimulando esses profissionais a notificarem os eventos adverso, a fim de analisá-los, corrigi-los e preveni-los (ARAÚJO JS, et al. 2016).

É notório que as fragilidades relacionadas à notificação de eventos adversos ainda permeiam a Segurança do paciente. O medo decorrente de uma cultura de segurança frágil e de uma gestão que aplica ações punitivas inviabiliza a notificação, fortalecendo a subnotificação.

\section{CONSIDERAÇÕES FINAIS}

A segurança do paciente, na atualidade, suscita melhorias atreladas aos processos de trabalho e é uma temática extremamente relevante em se tratando do âmbito hospitalar, visando sempre à prestação do cuidado de forma segura. Observou-se os avanços da notificação dos eventos adversos, visto que nos estudos abordados foi identificando a potencialidade dessa ferramenta na melhoria dos processos e instrumento fundamental para Segurança do Paciente na prestação dos cuidados. A notificação de eventos adversos já vem sendo apontada como uma medida preventiva para ocorrência de erros na assistência à saúde. Contudo, fragilidades ainda são identificadas, ressalta-se que a cultura de segurança ainda não é elemento predominante da mentalidade dos profissionais. O erro é um termo forte, pois dependendo da magnitude do mesmo o paciente será pouco ou muito prejudicado. Apesar dos avanços, as fragilidades relacionadas à notificação de eventos adversos ainda permeiam a Segurança do paciente. O medo decorrente de uma cultura de segurança frágil e aplicação de ações punitivas, inviabiliza a notificação e fortalece a subnotificação.

\section{REFERÊNCIAS}

1. ANVISA. Portaria no 529, de 1 de abril de 2013. Ministério da Saúde - MS. Agência Nacional de Vigilância Sanitária - Anvisa.

2. ARAÚJO JS, et al. Conhecimento dos enfermeiros sobre evento adverso e os desafios para a sua notificação. Revista Cogitare Enfermagem, 2016; 21 (4): 01-08.

3. CHAGAS KD, SELOW MLC. O enfermeiro frente à notificação de eventos adversos em busca de garantia de qualidade e segurança assistencial. Vitrine Prod. Acad., 2016; 4 (2): 89-161.

4. CAPUCHO HC, et al. Segurança do paciente: comparação entre notificações voluntárias manuscritas e informatizadas sobre incidentes em saúde. Revista Gaúcha de Enfermagem, 2013; 34 (1): 164-172. 
5. DUARTE SCM, et al . Eventos adversos e segurança na assistência de enfermagem. Rev. Bras. Enferm., 2015; 68 (1): 144-154.

6. FERNANDO FSL de, et al. Segurança do paciente: análise reflexiva. Revista de Enfermagem UFPE on line., 2016; 10 (2): 894-902.

7. MIRANDA AP, et al. Contribuição da enfermagem à Segurança do Paciente: Revisão integrativa. SANARE Sobral, 2017; 16(1): 109-117.

8. OLIVEIRA RM, et al. Estratégias para promover segurança do paciente: da identificação dos riscos às práticas baseadas em evidências. Esc. Anna Nery [online], 2014; 18(1): 122-129.

9. PAIVA MCMS, et al. Motivos da equipe de enfermagem para a notificação de eventos adversos. Revista Latino-Americana de Enfermagem, 2014; 22 (5): 747-754.

10. REIS CT, et al. Segurança do paciente como dimensão da qualidade do cuidado de saúde: um olhar sobre a literatura. Ciênc. saúde coletiva[online], 2013; 18 (7): 2029-2036.

11. SILVA AT, et al. Assistência de enfermagem e o enfoque da segurança do paciente no cenário brasileiro. Saúde debate, 2016; 40 (111): 292-301.

12. SIMAN AG, et al. A prática da notificação de eventos em um hospital de ensino. Rev. Esc. Enferm. USP, 2017; 51: e03243. 